\title{
An adaptive optics biomicroscope for mouse retinal imaging
}

\author{
David P. Biss ${ }^{a}$, Robert H. Webb ${ }^{a}$, Yaopeng Zhou ${ }^{b}$, Thomas G. Bifano ${ }^{b}$, Parisa Zamiri $^{c}$, and \\ Charles P. Lin ${ }^{c}$ \\ ${ }^{a}$ Schepens Eye Research Institute, 20 Staniford Street, Boston, MA, 02114, USA; \\ ${ }^{b}$ Boston University, 15 St. Mary Street, Brookline, MA, 02446, USA; \\ ${ }^{c}$ Wellman Center for Photomedicine, Harvard Medical School, \\ Massachusetts General Hospital, Boston, MA, 02114, USA
}

\begin{abstract}
In studying retinal disease on a microscopic level, in vivo imaging has allowed researchers to track disease progression in a single animal over time without sacrificing large numbers of animals for statistical studies. Historically, a drawback of in vivo retinal imaging, when compared to ex vivo imaging, is decreased image resolution due to aberrations present in the mouse eye.

Adaptive optics has successfully corrected phase aberrations introduced the eye in ophthalmic imaging in humans. We are using adaptive optics to correct for aberrations introduced by the mouse eye in hopes of achieving cellular resolution retinal images of mice in vivo. In addition to using a wavefront sensor to drive the adaptive optic element, we explore the using image data to correct for wavefront aberrations introduced by the mouse eye. Image data, in the form of the confocal detection pinhole intensity are used as the feedback mechanism to control the MEMS deformable mirror in the adaptive optics system. Correction for wavefront sensing and sensor-less adaptive optics systems are presented.
\end{abstract}

Keywords: Medical imaging, microscope, adaptive optics, MEMS, retinal imaging

\section{INTRODUCTION}

When studying the retinal disease progression on a microscopic scale in a mouse model, large mouse populations are sacrificed at different times during the disease cycle. This provides researchers with "snap-shots" of the disease's progress in multiple mice. Researchers are then forced to study disease by sacrificing large numbers of mice and using statistical analysis to determine the disease effects accurately over time. In vivo retinal imaging does not require sacrificing an animal to view its retina, and allows for tracking disease progression in the same mouse over the disease's timespan. While in vivo imaging overcomes the needs for statistical analyses and sacrificing larger mice numbers, aberrations in the mouse eye limit the best possible resolution attainable when compared to ex vivo imaging. ${ }^{1-4}$ An example of the differences between ex vivo and in vivo retinal images is in Fig. 1. Fig. 1 is an image set of microglia cells labeled with green fluorescent protein $(\mathrm{GFP}+)$ in the mouse retina. Fig. 1(a) is an ex vivo flatmount image of the retina, while fig. 1(b) is an in vivo retinal image taken with the same imaging instrument. In Fig. 1(a) the dendrites of microglia cells (approximately $1 \mu m$ wide) branching off from the microglia cell body, but in Fig. 1 the dendrites are not easily resolved.

In confocal imaging, aberrations will blur the light focused at the confocal pinhole and cause reduced image intensity and resolution. Adaptive optics has been utilized in ophthalmic imaging to overcome aberrations introduced by the eye in humans and primates to provide near-diffraction limited imaging. ${ }^{5-12}$ While most AO systems rely on a wavefront sensor, there has been extensive work in microscopy on so-called sensor-less AO systems. ${ }^{13-15}$ By devising an algorithm to configure the adaptive element one can maximize the amount of light passing through the confocal pinhole and correct the aberrations in the optical system. In the case of confocal microscopes, these sensor-less AO systems use the confocal pinhole intensity as a metric and formulate

Further author information: (Send correspondence to David P. Biss)

David P. Biss.: E-mail: david.biss@schepens.harvard.edu, Telephone: 16179122503

Stephen A. Burns' current affiliation is with Indiana University, School of Optometry, 800 E. Atwater Ave., Bllomington, IN, 47405, USA

MEMS Adaptive Optics, edited by Scot S. Olivier, Thomas G. Bifano, Joel A. Kubby, Proc. of SPIE Vol. 6467, 646703, (2007) · 0277-786X/07/\$18 · doi: 10.1117/12.707531 


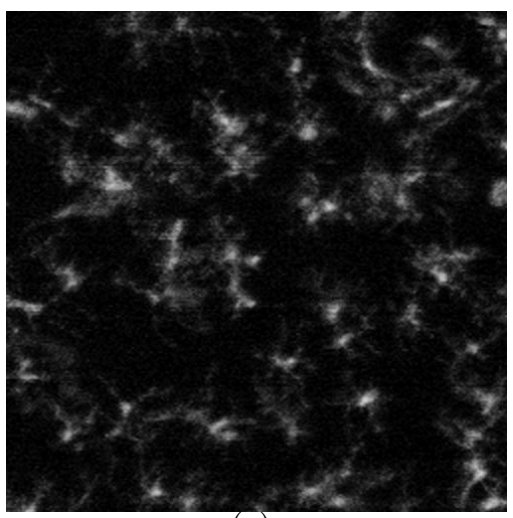

(a)

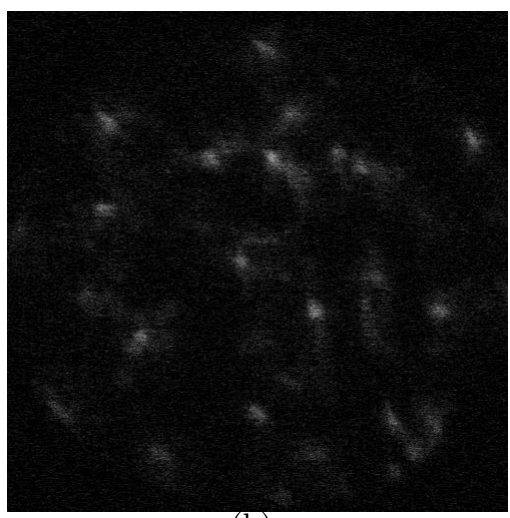

(b)

Figure 1. GFP+ microglia in the retina. (a) ex vivo flatmount image. (b) in vivo image with a biomicroscope. Both images have their pixel magnitudes normalized to the maximum pixel value.

a optimization algorithm for the adaptive element to maximize the pinhole intensity. This sensor-less AO system has also be proposed for ophthalmic imaging. ${ }^{11}$

In these Proceedings, we present two AO biomicroscope systems for retinal imaging in vivo. One system is a wavefront sensing AO system that uses a Shack Hartmann Wavefront Sensor (SHWS) to monitor the fluorescent wavefront from the mouse retina. A second system is a sensor-less system that monitors the confocal pinhole signal and uses an algorithm to optimize the adaptive optic. In both systems the adaptive optic is a deformable MEMS mirror.

\section{METHODS}

\subsection{Biomicroscope}

The biomicroscope is a video-rate (30 fps) imaging system that relies on a polygon scanner and a galvanometric mirror to generate the raster for the image. It has two imaging channels, a fluorescent channel, and a reflectance channel and has three laser wavelengths for fluorescence excitation. Fig. 2 is a schematic of the biomicroscope. A $635 \mathrm{~nm}$ laser is used to excite Evans Blue fluorescence (Micro Laser Systems, Garden Grove, CA, USA). A 491 nm laser is used to excite GFP + cells (Dual Calypso, Colbolt AB, Stockholm, Sweden), this laser has a second wavelength at $532 \mathrm{~nm}$ for other fluorophore excitation. An entrance pupil is formed at the source and imaged onto the AO element. The AO element is a deformable MEMS mirror (DM) (Boston Micromachines Corp., Watertown, MA, USA). The mirror consists of a square array of 144 actuators with a maximum stroke of $3.5 \mu m$. The mirror was placed in a "two-pass" position, so that both the excitation and the fluorescent beams were reflected by the mirror. The pupil at the DM is imaged on a 36 facet polygon (Lincoln Laser Corp., Phoenix, AZ, USA) that rotates at 480 revolutions per second and provides the horizontal raster for the image. The pupil at the polygon is relayed to a galvanometric mirror (GSI Lumonics, Billerica, MA, USA), that provides the vertical scan for the image. This pupil is in turn imaged at the entrance pupil of a $50 \mathrm{x}$, $0.5 \mathrm{NA}$ long working distance $(13 \mathrm{~mm})$ microscope objective (Mitutoyo Corporation, Kanagawa, Japan). The mouse was mounted on a translation stage to position the retina into the microscope's focal plane. Fluorescence is collected by the microscope objective and propagates back through the system. After the fluorescent light is reflected off the DM it is directed into a detection arm by a dichroic mirror. A beamsplitter reflects some of that light to be focused through a confocal pinhole $(100 \mu \mathrm{m}$ diameter, that is $10 \mu \mathrm{m}$ imaged on to the retina) and detected by a PMT (R3896, Hamamatsu Photonics K. K., Hamamatsu City, Japan) while the transmitted light is collected by the Shack Hartmann wavefront sensor (SHWS). 


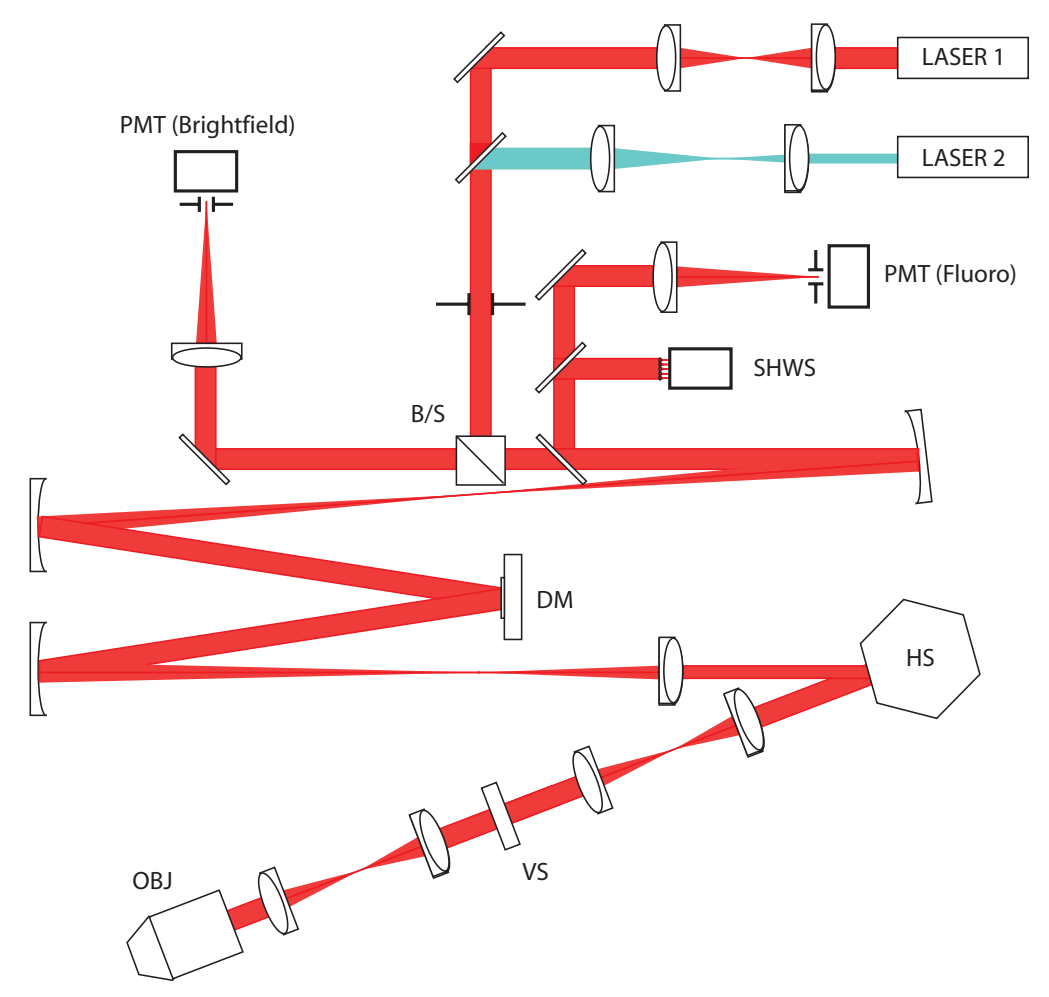

Figure 2. Biomicroscope. LASER 1 is a $635 \mathrm{~nm}$ laser source. LASER 2 is a $491 \mathrm{~nm}$ laser source. B/S is a beamsplitter. DM is a deformable mirror (adaptive element). HS is a polygon that provides the horizontal scan. VS is a galvanometric mirror for the vertical scan. OBJ is a long working distance microscope objective. SHWS is a Shack Hartmann Wavefront Sensor. PMT (Fluoro) is the confocal fluorescence detection channel. PMT (Brightfield) is the confocal brightfield detection channel.

\subsection{Wavefront Sensing Adaptive Optics}

The wavefront sensing AO system consists of a SHWS for wavefront measurement and a DM as the adaptive element. The SHWS, consisting of a CCD camera (UNIQ, Santa Clara, CA, USA) and a lenslet array (Adaptive Optics Associates, Cambridge, MA, USA), measures the wavefront of the fluorescent light at a plane conjugate to both the deformable mirror and the pupil of the microscope objective. A simple scattering target (here a piece of paper) in the focal plane of the microscope objective generates a calibration image in the SHWS. From this calibration image the control software builds an influence matrix between the action of individual DM actuators and the resulting SHWS pattern. The algorithm measures the SHWS spot deviation from the calibration image, and then adjusts the mirror to minimize them. While the SHWS measures the wavefront of the fluorescent light, the DM corrects the wavefront of both the forward and backward propagating beams. Thus, the two-pass design corrects the illumination and fluorescence wavefront.

\subsection{Sensor-less Adaptive Optics}

The sensor-less AO system uses an image to generate the metric for the optimization algorithm. If the image signal to noise is low, frame averaging can be used to reduce noise in the metric. In these experiments the metric used was the mean pixel intensity. On the mirror the amplitude of the first 35 Zernike polynomials were optimized. ${ }^{16}$ A simple random walk algorithm was used to maximize the metric.

\subsection{Animal Handling}

$\mathrm{BALB} / \mathrm{c}, \mathrm{C} 57 \mathrm{BL} / 6$ or B6.129P-Cx3cr $1^{\text {tm1Litt }} / \mathrm{J}$ mice were anesthetized using a mixture of ketamine and xylazine, in order to obtain long series of images and to facilitate handling the mice. All experimental procedures conformed 
to the ARVO Statement for the Use of Animals for Ophthalmic and Vision Research, and were approved by the Massachusetts General Hospital (SRAC). Resident glial cells of the retina, microglia, express GFP in the

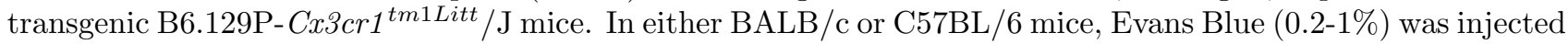
to label the vasculature. All mice received a drop of tropicamide $1 \%$ to dilate the pupil. The mice were placed on a heated microscope stage and their eyes were covered with a lubricating agent (Methocel). A glass cover slip was positioned on the anterior surface of the cornea for visualization. The combination of the flat cover slip and microscope objective prevented the cornea from drying and nulled the refractive power of the cornea. Power levels of $1 \mathrm{~mW}$ and $0.2 \mathrm{~mW}$ at $635 \mathrm{~nm}$ and $491 \mathrm{~nm}$, respectively, entered the eye. We estimated that our field of view is $0.5 \mathrm{~mm}$ square. These values are below the maximum permissible exposure for humans with exposure times less than 3 minutes. ${ }^{17}$

\section{EXPERIMENTS}

\subsection{Wavefront Sensing Adaptive Optics}

In vivo images of Evans Blue labeled blood vesssels are shown in Fig. 3 along with measured SHWS spot shifts superimposed on the calculated wavefront and a intensity plot along a segment of the images. The images shown are averages of 30 frames that are co-registered before averaging. To compute the wavefront from the measured spot displacements Zernike coefficients were fitted to the displacements. ${ }^{16}$ The effect of the adaptive correction can be seen when comparing Fig. 3(a) and (b). The calculated wavefront with the measured SHWS spot shifts for the uncorrected and corrected images are shown in Fig. 3(c) and (d), respectively. Fig. 3(e) is a plot of the intensity of Fig. 3(a) and (b) along the white line shown. The blue dashed line is the intensity plot for (a) and the solid green line is the intensity plot for (b).

\subsection{Sensor-less Adaptive Optics}

Fig. 4 shows in vivo images of Evans Blue labeled retinal vessels with the sensorless AO system on and off, the final mirror deflections after 100 iterations of the correction algorithm, and intensity line plots from the in vivo images. The images shown are 30 averaged frames that are co-registered prior to averaging. In Fig. 4(c) the final mirror position at the end of the optimization cycle is shown. Fig. 4(d) is an intensity plot from the white lines in (a) and (b). The blue dashed line corresponds to Fig. 4(a) and the green solid line corresponds to Fig. $4(\mathrm{~b})$.

\section{RESULTS}

It would be desirable in the wavefront sensing situation to measure the wavefront for $\mathrm{AO}$ correction using backscattered light, but this proved difficult in practice. This difficulty probably arises because the SHWS lenslets have a very high f-number and therefore detect the light from all retinal layers and the posterior surface of the lens, that is quite close to the retina of the mouse eye. Unlike the human eye, the mouse retina is thick $(200 \mu \mathrm{m})$ relative to its focal length (approx. $2 \mathrm{~mm}$ ) and these reflections represent a mix of aberrations, as well as very large blur circles. For the fluorescently labeled cells, the source for both the SHWS measurements and the resulting confocal image is localized to specific layers and cells.

In both the wavefront sensing and sensor-less $\mathrm{AO}$ situations correction is achieved. With the $\mathrm{AO}$ systems correcting, the brightness and resolution are increased. Table 1 lists the maximum pixel value, mean pixel value, and the Michelson contrast for the images in Fig. 3 and Fig. 4. In Figs. 3 and 4 the AO system helps resolve capillaries and make larger vessels sharper. The contrast in Fig. 3 is increased by 6\%. The contrast in Fig. 4 is increased by $35 \%$.

With the wavefront AO correction running, the RMS spot deviation of the SHWS image is reduced from $11.19 \mu \mathrm{m}$ to $5.09 \mu \mathrm{m}$. In the data provided for Fig. 3 the system did not provide full correction, meaning the RMS spot deviation was reduced to zero, since the mirror stroke was saturated. Fig. 3 provides evidence of the increase in contrast with the $\mathrm{AO}$ correction running.

For the sensor-less AO system the mirror stroke was not saturated. By looking at Fig. 4(c) we can infer the approximate shape of the wavefront error in the system; in this case the dominant aberration looks to represent 


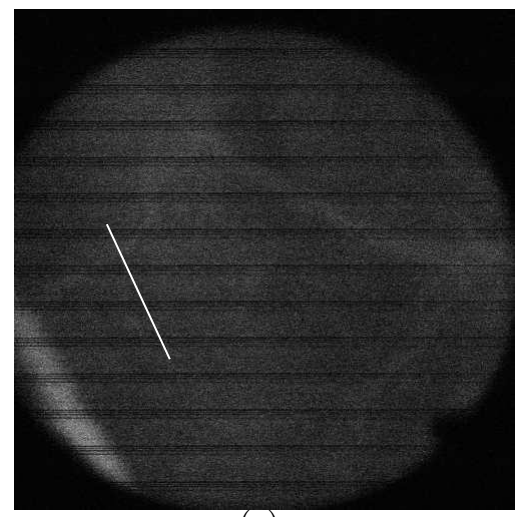

(a)

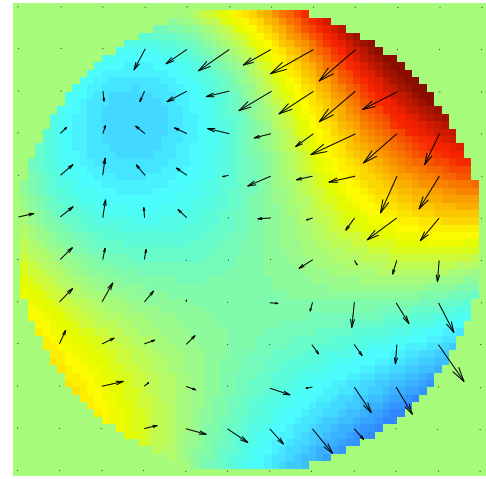

(c)

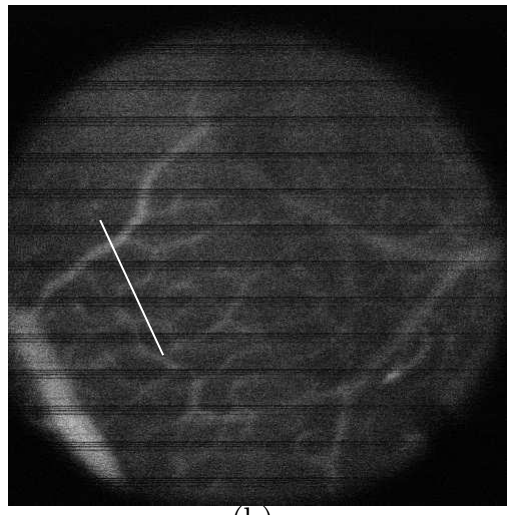

(b)

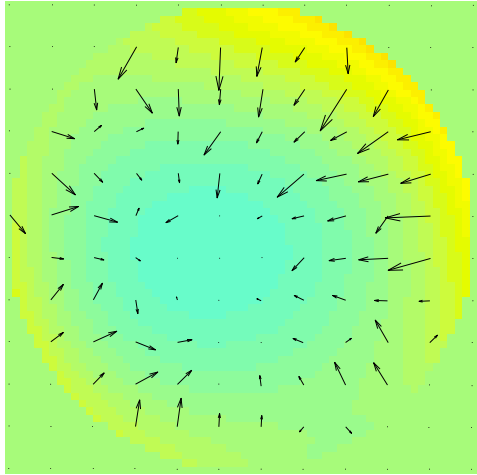

(d)

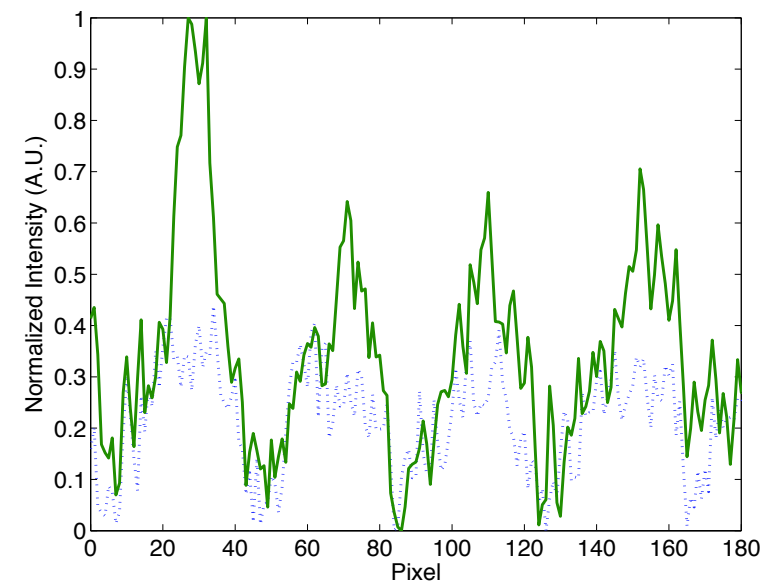

(e)

Figure 3. In vivo retinal vessels labeled with Evans Blue and measured wavefront. (a) aberrated image. (b) image corrected with adaptive optics. (c) measured wavefront corresponding to the aberrated image. (d) measured wavefront with adaptive optic system on. (d) Line plot comparing image with and without AO correction. 


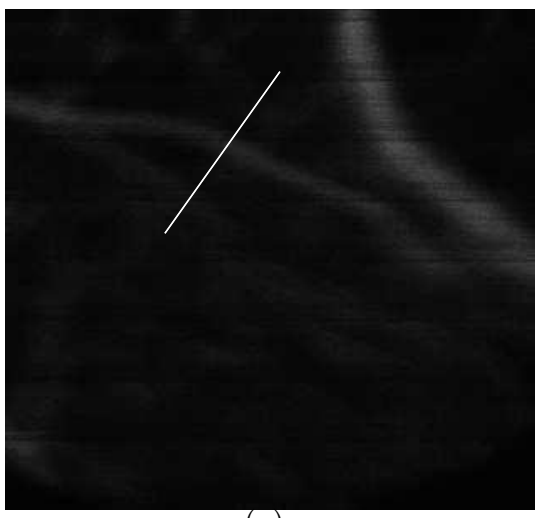

(a)

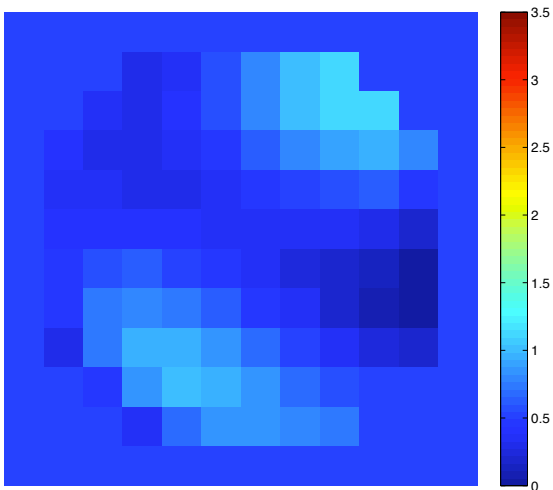

(c)

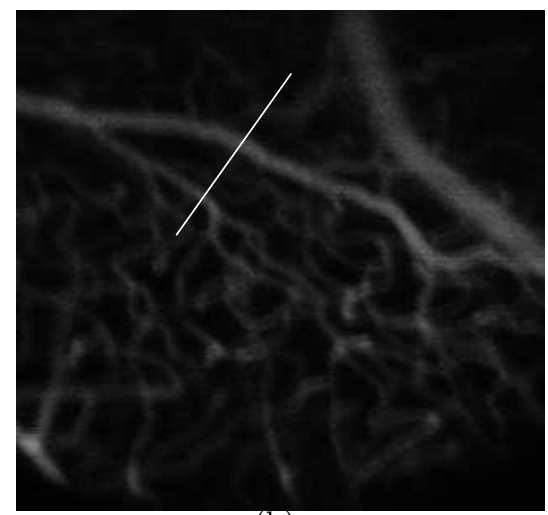

(b)

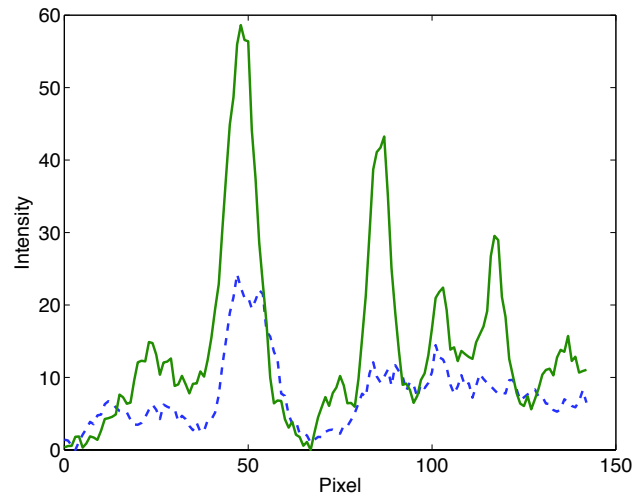

(d)

Figure 4. In vivo images of retinal vessels labeled with Evans Blue. (a) aberrated image with the contrast adjusted for visualization. (b) image corrected with sensorless AO system with the contrast adjusted for visualization. (c) image of the DM actuator deflections at the end of the optimization cycle. (d) image intensity plot from (a) and (b). 
Table 1. Measured image attributes.

\begin{tabular}{|c|c|c|c|}
\hline Image & Mean pixel value & Maximum pixel value & Contrast \\
\hline \hline Fig. 3(a) & 9328 & 45181 & 4.791 \\
\hline Fig. 3(b) & 10630 & 54493 & 5.084 \\
\hline \hline Fig. 4(a) & 9.956 & 90 & 8.839 \\
\hline Fig. 4(b) & 11.622 & 139 & 11.960 \\
\hline
\end{tabular}

astigmatism. In Fig. 4(d) if we examine the full-width at half-maximum (FWHM) of the dominant peak we can compare the resolution with and without correction. With correction on, the peak has a FWHM of 10 pixels, and with the correction off the peak has a FWHM of 15 pixels indicating increased resolution with AO correction.

While both systems can correct aberrations, the sensor-less system requires longer periods to optimize the DM. A more suitable search algorithm, such as the stochastic parallel-gradient-descent algorithm, could reduce the time to optimize the DM shape. ${ }^{18}$ This type of algorithm is less susceptible to local minima and tends to converge faster than a random walk. Another alternative algorithm is based on sphere packing and has been proposed by Booth for sensor-less AO systems. ${ }^{15}$ This type of correction could be quite valuable for faster optimization schemes, provided that certain caveats are met. Beyond better algorithms, choosing a more appropriate image metric would help the optimization.

\section{CONCLUSION}

We have demonstrated two AO systems for imaging the in vivo mouse retina. Both wavefront sensing and sensor-less AO systems have been used to correct for aberrations. With these AO systems we have been able to visualize fluoresently labeled capillaries. In some cases only partial correction was achieved. In these cases, further improvement will require a deformable mirror with a longer stroke, or an external doubler. ${ }^{19}$ While the sensor-less system did correct aberrations, the time to optimize the mirror was longer when compared to the wavefront sensing AO system. With better algorithms, such as the algorithm proposed by Booth, ${ }^{15}$ faster optimization times could be achieved. Without the need to divert fluorescent signal to the wavefront sensor the sensor-less system would have higher sensitivity than its wavefront sensing counterpart. The sensor-less system is also a simpler optical design, which could prove useful for devices to be used by non-technical personnel. Nevertheless, both systems show marked improvement in imaging.

The ability to visualize the retina at the cellular level is a great tool for studying the role of inflammation and/or apoptosis in the pathogenesis of diabetic retinopathy and age related macular degeneration, diseases with increasing incidence and morbidity. In vivo imaging would allow the temporal study of therapies in the same animal and would greatly enhance new therapeutic strategies.

\section{ACKNOWLEDGMENTS}

This research was supported by NIH grant EY-14106 and partial support for software development was also provided by NIH grant EY-014375.

\section{REFERENCES}

1. C. Schmucker and F. Schaeffel, "A paraxial schematic eye model for the growing C57BL/6 mouse," Vision Res. 44, pp. 1857 - 1867, 2004.

2. E. G. de la Cera, G. Rodríguez, L. Llorente, F. Schaeffel, and S. Marcos, "Optical aberrations in the mouse eye," Vision Res. 46, pp. 2546-2553, 2006.

3. M. Paques, M. Simonutti, M. J. Roux, S. Picaud, E. Levavasseur, C. Bellman, and J. A. Sahel, "High resolution fundus imaging by confocal scanning laser ophthalmoscopy in the mouse," Vision Res. 46, pp. 13361345, 2006. 
4. T. Higashide, I. Kawaguchi, S. Ohkubo, H. Takeda, and K. Sugiyama, "In vivo imaging and counting of rat retinal ganglion cells using a scanning laser ophthalmoscope," Invest. Ophthalmol. and Vis. Sci. 47, pp. 2943-, 2006.

5. J. Liang, D. R. Williams, and D. T. Miller, "Supernormal vision and high-resolution retinal imaging through adaptive optics," J. Opt. Soc. Am. A 14, pp. 2884-, 1997.

6. A. Roorda, F. Romero-Borja, W. J. D. III, H. Queener, T. J. Hebert, and M. C. Campbell, "Adaptive optics scanning laser ophthalmoscopy," Opt. Express 10, pp. 405-412, 2002.

7. N. Doble, G. Yoon, L. Chen, P. Bierden, B. Singer, S. Oliver, and D. R. Williams, "Use of a microelectromechanical mirror for adaptive optics in the human eye," Opt. Lett. 27, pp. 1537-1539, 2002.

8. D. X. Hammer, R. D. Ferguson, C. E. Bigelow, N. V. Iftimia, T. E. Ustun, and S. A. Burns, "Adaptive optics scanning laser ophthalmoscope for stabilized retinal imaging," Opt. Express 14, pp. 3354-3367, 2006.

9. Y. Zhang, S. Poonja, and A. Roorda, "Mems-bases adaptive optics scanning laser ophthalmoscopy," Opt. Lett. 31, pp. 1268-1270, 2006.

10. D. C. Gray, W. Merigan, J. I. Wolfing, B. P. Gee, J. Porter, A. Dubra, T. H. Twietmeyer, K. Ahamd, R. Tumbar, F. Reinholz, and D. R. Williams, "In vivo fluorescence imaging of primate retinal ganglion cells and retinal pigment epithelial cells," Opt. Express 14, pp. 7144-6158, 2006.

11. S. Zommer, E. N. Ribak, S. G. Lipson, and J. Adler, "Simulated annealing in ocular adaptive optics," Opt. Lett. 31, pp. 939-941, 2006.

12. K. Grieve, P. Tiruveedhula, Y. Zhang, and A. Roorda, "Multi-wavelength imaging with the adaptive optics scanning laser ophthalmoscope," Opt. Express 14, pp. 12230-12242, 2006.

13. M. J. Booth, M. A. A. Neil, R. Juškaitis, and T. Wilson, "Adaptive aberration correction in a confocal microscope," Proc. Nat. Acad. of Sci. 99, pp. 5788-5792, 2002.

14. L. Sherman, J. Y. Ye, O. Albert, and T. B. Norris, "Adaptive correction of depth-induced aberrations in multiphoton scanning microscopy using a deformable mirror," J. of Microscopy 206, pp. 65-71, 2002.

15. M. Booth, "Wave front sensor-less adaptive optics: a model-based approach using sphere packings," Opt. Express 14, pp. 1339-1352, 2006.

16. L. N. Thibos, R. A. Applegate, J. T. Schwiegerling, R. H. Webb, and VISA Standards Taskforce Members, "Standards for reporting the optical aberrations of eyes," in Vision Science and its Applications, V. Lakshminarayan, ed., OSA Trends in Optics $\&$ Photonics 35, pp. 232-244, 2000.

17. F. C. Delori, R. H. Webb, and D. H. Sliney, "Maximum permissible exposures for ocular safety (ANSI 2000), with emphasis on ophthalmic devices," J. Opt. Soc. Am. A in press, 2007.

18. M. A. Vorontsov and V. P. Sivokon, "Stochastic parallel-gradient-descent technique for hgih-resolution wave-front phase-distortion correction," J. Opt. Soc. Am. A 15, pp. 2745-2758, 1998.

19. R. H. Webb, M. J. Albanese, Y. Zhou, T. Bifano, and S. A. Burns, "Stroke amplifier for deformable mirrors," Appl. Opt. 43, pp. 5330-5333, 2004. 